\title{
Hyeonggaeyeongyo-Tang for Treatment of Allergic and Nonallergic Rhinitis: A Prospective, Nonrandomized, Pre-Post Study
}

\author{
Min-Hee Kim, ${ }^{1,2}$ Jaewoong Son, ${ }^{1,2}$ Hae Jeong Nam, ${ }^{3}$ Seong-Gyu Ko, ${ }^{4}$ and Inhwa Choi ${ }^{2,3}$ \\ ${ }^{1}$ Department of Clinical Korean Medicine, Graduate School, Kyung Hee University, Seoul, Republic of Korea \\ ${ }^{2}$ Department of Otorhinolaryngology of Korean Medicine, Kyung Hee University Hospital at Gangdong, Seoul, Republic of Korea \\ ${ }^{3}$ Department of Ophthalmology \& Otorhinolaryngology, College of Korean Medicine, Kyung Hee University, Seoul, Republic of Korea \\ ${ }^{4}$ Department of Preventive Medicine, College of Korean Medicine, Kyung Hee University, Seoul, Republic of Korea
}

Correspondence should be addressed to Seong-Gyu Ko; epiko@khu.ac.kr and Inhwa Choi; inhwajun@hanmail.net

Received 27 May 2016; Revised 27 July 2016; Accepted 7 August 2016

Academic Editor: Xiang Liu

Copyright (c) 2016 Min-Hee Kim et al. This is an open access article distributed under the Creative Commons Attribution License, which permits unrestricted use, distribution, and reproduction in any medium, provided the original work is properly cited.

\begin{abstract}
Hyeonggaeyeongyo-tang (HYT) is an ancient formula of oriental medicine traditionally used to treat rhinitis; however, clinical evidence has not yet been established. The aim of this study was to investigate the short-term and long-term efficacy and safety of HYT for chronic rhinitis. Adult subjects with chronic rhinitis symptoms were recruited. The subjects received HYT for 4 weeks and had follow-up period of 8 weeks. Any medicines used to treat nasal symptoms were not permitted during the study. The skin prick test was performed to distinguish the subjects with allergic rhinitis from those with nonallergic rhinitis. After treatment, the total nasal symptoms score and the Rhinoconjunctivitis Quality of Life Questionnaire score significantly improved in the whole subject group, in the allergic rhinitis group, and in the nonallergic rhinitis group, with no adverse events. This improvement lasted during a follow-up period of 8 weeks. Total IgE and eosinophil levels showed no significant difference after treatment in the allergic rhinitis group. HYT improved nasal symptoms and quality of life in patients with allergic rhinitis and nonallergic rhinitis. This is the first clinical study to evaluate the use of HYT to treat patients with rhinitis. This trial has been registered with the ClinicalTrials.gov Identifier NCT02477293.
\end{abstract}

\section{Introduction}

Rhinitis is defined by its clinical symptoms: rhinorrhea, nasal congestion, nasal itching, and sneezing. Anywhere from 10 to $40 \%$ of the population in industrialized countries has rhinitis based on epidemiologic surveys [1-4]. Chronic rhinitis (CR) is a chronic form of rhinitis and has been historically divided into allergic rhinitis (AR) and nonallergic rhinitis (NAR).

In Korea, several herbal medicines have been used to treat rhinitis. Hyeonggaeyeongyo-tang (HYT), also known as Keigai-rengyo-to or Jing Jie Lian Qiao Tang, is mixed herbal formula that has been used for hundreds of years in the treatment of rhinitis, rhinosinusitis, and acne. In an exploratory study, HYT was ranked first on the list of most commonly used herbal medications for treatment of allergic rhinitis in three Korean medical hospitals [5]. In another study, specialists in the Department of Otorhinolaryngology of Korean Medicine selected HYT as the third most preferred medicine to treat allergic rhinitis [6].

In animal studies, HYT has been shown to decrease the vascular permeability response to intradermal histamine and serotonin and to suppress a delayed type hypersensitivity response $[7,8]$. Park and Hong reported that HYT has antiinflammation effects for $\mathrm{AR}$ via the suppression of NF- $\kappa \mathrm{B}$ activation and iNOS production in BALB/c mice [9]. Hong et al. reported that HYT reduces infiltration of inflammatory cells and mast cells into the nasal cavity and reduces the levels of cytokines and leukocytes in the blood in an ovalbumininduced AR model [10]. In a clinical study, HYT has shown effectiveness in the treatment of adult patients with acne 
TABLE 1: Study's flow chart.

\begin{tabular}{|c|c|c|c|c|c|c|}
\hline \multirow{2}{*}{$\begin{array}{l}\text { Stage } \\
\text { Visit }\end{array}$} & \multirow{2}{*}{$\begin{array}{c}\text { Screening } \\
1\end{array}$} & \multicolumn{3}{|c|}{ Active treatment (4-week) } & \multicolumn{2}{|c|}{ Follow-up (8-week) } \\
\hline & & 2 & 3 & 4 & 5 & 6 \\
\hline Weeks & -1 & 0 & 2 & 4 & 8 & 12 \\
\hline Informed consent & O & & & & & \\
\hline \multicolumn{7}{|l|}{ Inclusion/exclusion criteria } \\
\hline Skin prick test & & O & & & & \\
\hline TNSS \& RQLQ & & O & O & O & O & O \\
\hline Total IgE \& eosinophil count ${ }^{\dagger}$ & & $\mathrm{O}$ & & $\bigcirc$ & & \\
\hline Vital sign & O & O & O & $\bigcirc$ & O & $\bigcirc$ \\
\hline Laboratory tests for safety assessment ${ }^{*}$ & O & & & O & & \\
\hline Adverse event & & & O & O & O & O \\
\hline
\end{tabular}

vulgaris [11]. To our knowledge, no other clinical studies have evaluated HYT for nasal symptoms thus far.

We conducted a prospective, nonrandomized, singlearmed, pre-post study of Korean adults with rhinitis. The aim of this study was to investigate the short-term and longterm efficacy and the safety of HYT treatment for allergic and nonallergic rhinitis.

\section{Methods}

2.1. Study Design. This prospective, nonrandomized, singlearmed, pre-post study was conducted at the Department of Otorhinolaryngology of Korean Medicine at Kyung Hee University Hospital at Gangdong. The study's flow chart is shown in Table 1 . This study was approved by the Institutional Review Board of Kyung Hee University Hospital at Gangdong (KHNMC-OH-IRB 2015-04-009). Written informed consent was obtained from all subjects prior to enrollment.

2.2. Subjects. A total of 40 subjects with CR were enrolled. The inclusion criteria were as follows: (1) age of 18-65 years, (2) presence of one or more nasal symptoms (rhinorrhea, nasal congestion, nasal itching, and sneezing) for more than 12 weeks, and (3) moderate-to-severe rhinitis (at least one of the following moderate abnormality conditions: sleep disturbance; limitations in daily activity, physical exercise, or leisure activity; work/school limitations; and discomfort from several symptoms) [12]. The exclusion criteria were as follows: (1) treatment with nasal/oral corticosteroids within the past month; nasal cromolyn or tricyclic antidepressants within the past two weeks; or nasal/oral decongestants, nasal/oral antihistamines, or antileukotrienes within the past week, (2) presence of hypertension (systolic $\geq 180 \mathrm{mmHg}$ or diastolic $\geq$ $100 \mathrm{mmHg}$ ) or severe anemia (hemoglobin $\leq 10 \mathrm{~g} / \mathrm{dL}$ (male) and $9 \mathrm{~g} / \mathrm{dL}$ (female)), (3) presence of abnormal liver function (aspartate transaminase (AST) or alanine transaminase $(\mathrm{ALT}) \geq 100 \mathrm{IU} / \mathrm{L})$ or abnormal renal function (blood urea nitrogen $(\mathrm{BUN}) \geq 30 \mathrm{mg} / \mathrm{dL}$ or creatinine $\geq 1.8 \mathrm{mg} / \mathrm{dL}$ (male) and $1.5 \mathrm{mg} / \mathrm{dL}$ (female)), (4) presence of neoplasm, severe
TABLE 2: The composition of herbal medicines in Hyeonggaeyeongyo-tang.

\begin{tabular}{lc}
\hline Component & Volume (g) \\
\hline Schizonepeta tenuifolia & 0.50 \\
Forsythia fruit & 0.50 \\
Saposhnikovia radix & 0.50 \\
Angelicae gigantis radix & 0.50 \\
Cnidii rhizome & 0.50 \\
Paeoniae radix alba & 0.50 \\
Glycyrrhizae radix & 0.50 \\
Bupleuri radix & 0.50 \\
Ponciri fruit & 0.50 \\
Scutellariae radix & 0.50 \\
Gardeniae fruit & 0.50 \\
Angelicae dahuricae radix & 0.83 \\
Platycodi radix & 0.83 \\
\hline
\end{tabular}

systemic inflammation, or other systemic diseases that affect rhinitis, (5) history of drug allergy, (6) history of anaphylaxis for allergic tests, and (7) pregnancy or lactation.

2.3. Study Drug. Patients were treated with HYT extracted with water (Hanpoong Hyeonggaeyeongyo-tang granules, Hanpoong Pharm \& Food Co., Ltd., Jeonju, Republic of Korea). It is a brown, bitter, herbal extract and was produced according to the Korean Good Manufacturing Practice (GMP) guidelines as permitted by the Korean Food \& Drug Administration. HYT granules were sealed in opaque aluminum bags and administered to participants at doses of $3 \mathrm{~g}$ in accordance with standard guidelines for herbal prescription administration. The pharmacists instructed the subjects to dissolve HYT ( $3 \mathrm{~g}$ ) from each package in water and take the solutions 30 minutes after each meal three times per day for 4 weeks. The composition of HYT is shown in Table 2. During the study, any medications that may have affected 
nasal symptoms (antihistamines, corticosteroids, anticholinergics, antileukotriene drugs, decongestants, tricyclic antidepressants, phenothiazines, nonsteroidal anti-inflammatory drugs, or Korean herbal medicines that were judged by the researchers to affect nasal symptoms) were not permitted. Subjects that reported using any of these medications were excluded from the study.

2.4. Allergic Skin Prick Test. The skin prick test was performed according to routine procedure. Eleven common aeroallergens (Dermatophagoides farinae, Dermatophagoides pteronyssinus, dog fur, cat fur, grass mixture, tree mixture, mugwort, ragweed, Alternaria tenuis, Aspergillus fumigatus, and cockroach), negative controls (50\% glycerin saline), and positive controls ( $0.1 \%$ histamine phosphate) were used (Allergopharma GmbH \& Co. KG, Reinbek, Germany). The subjects who showed a positive reaction to the skin prick test were identified as AR, while subjects who showed no reaction to the skin prick test were identified as NAR.

2.5. Efficacy Assessment. Any change in the TNSS was considered the primary efficacy variable, while any change in the RQLQ was considered a secondary efficacy variable. The TNSS evaluates symptoms of rhinorrhea, nasal congestion, nasal itching, and sneezing on a 4-point scale. The total score range is from 0 to 12 , where $0=$ no symptoms, $1=$ mild symptom(s) (present but bearable), $2=$ moderate $\operatorname{symptom}(\mathrm{s})$ (present and uncomfortable), and 3 = severe symptom(s) (unbearable). Before and after medication we examined total serum IgE and eosinophil count for subjects with AR.

2.6. Safety Assessment. Before and after medication we assessed levels of AST/ALT, BUN/creatinine, complete blood counts (CBC) including white blood cell (WBC), red blood cell (RBC), hemoglobin, hematocrit, and platelet, and erythrocyte sedimentation rate (ESR) to ensure the subjects' safety. Throughout the trial all adverse events were noted in subjects' reports or in the case report forms.

2.7. Statistical Analysis. All statistical analyses were performed using the SPSS 21 (IBM Inc., Armonk, NY, USA), and values are presented as means \pm standard deviations. A repeated-measures ANOVA test with Bonferroni post hoc test was performed to evaluate the changes of TNSS and RQLQ scores throughout the 12 weeks. Independent $t$-tests were performed in order to analyze the intergroup analysis at each period. Paired $t$-tests were used to compare values before and after medication in each group. In all tests, a value of $p<0.05$ was considered statistically significant.

\section{Results}

3.1. Subjects. A total of 47 patients were screened, and 40 subjects with CR were included in this study. Seven patients were excluded because they had mild nasal symptoms. During the active treatment period, 2 subjects withdrew from
TABLE 3: Demographic characteristics of the study population.

\begin{tabular}{lc}
\hline Number of subjects & 40 \\
Male/female ( $n$ ) & $15 / 25$ \\
Age (mean \pm SD, years) & $38.68 \pm 12.86$ \\
Dropouts & \\
$\quad$ Personal reasons & 2 \\
$\quad$ Use of other medications & 3 \\
AR/NAR group ( $n$ ) & $16 / 24$ \\
Persistent allergens & \\
Dermatophagoides farinae & 15 \\
Dermatophagoides pteronyssinus & 15 \\
Cockroach & 1 \\
Dog fur & 0 \\
Cat fur & 5 \\
Aspergillus fumigatus & 2 \\
Alternaria tenuis & 3 \\
Seasonal allergens & \\
Grass mixture & \\
Mugwort & 1 \\
Tree mixture & 6 \\
Ragweed & 3 \\
\hline
\end{tabular}

SD, standard deviations; AR, allergic rhinitis; NAR, nonallergic rhinitis.

TABLE 4: Mean TNSS from baseline to week 12.

\begin{tabular}{lcccc}
\hline & Week 0 & Week 4 & Week 8 & Week 12 \\
\hline All subjects & $7.53 \pm 1.95$ & $5.10 \pm 2.81^{*}$ & $5.63 \pm 2.57^{*}$ & $5.43 \pm 3.09^{*}$ \\
AR & $8.31 \pm 1.58$ & $5.50 \pm 3.29^{*}$ & $6.19 \pm 3.08^{*}$ & $6.00 \pm 3.44^{*}$ \\
NAR & $7.00 \pm 2.02$ & $4.83 \pm 2.48^{*}$ & $5.25 \pm 2.15^{*}$ & $5.04 \pm 2.84^{*}$ \\
\hline
\end{tabular}

Mean \pm standard deviation, repeated-measures ANOVA test, and Bonferroni post hoc test.

${ }^{*}$ Significant difference $(p<0.05)$ compared with week 0 (baseline) in each group (no significant difference between week 4 , week 8 , and week 12 in each group).

TNSS, total nasal symptom score; AR, allergic rhinitis; NAR, nonallergic rhinitis.

the study because of personal reasons. During the followup period, 3 subjects were excluded because they used other medicines. There were 16 subjects who were identified as AR after skin prick test. All AR subjects showed positive reaction to at least one or more persistent allergens. There were no significant differences in sex, age, baseline TNSS, each nasal symptom score, and baseline RQLQ score between the AR group and the NAR group (Table 3 ).

3.2. TNSS. A statistically significant decrease in TNSS was observed after medication (week 4), and this decrease lasted for the follow-up period in the whole subject group (CR), the AR group, and the NAR group. There was no significant between-group difference at any time point (Table 4 and Figures 1(a) and 1(c)). All nasal symptoms, rhinorrhea, nasal congestion, nasal itching, and sneezing, showed significant improvement during treatment. There was no significant 


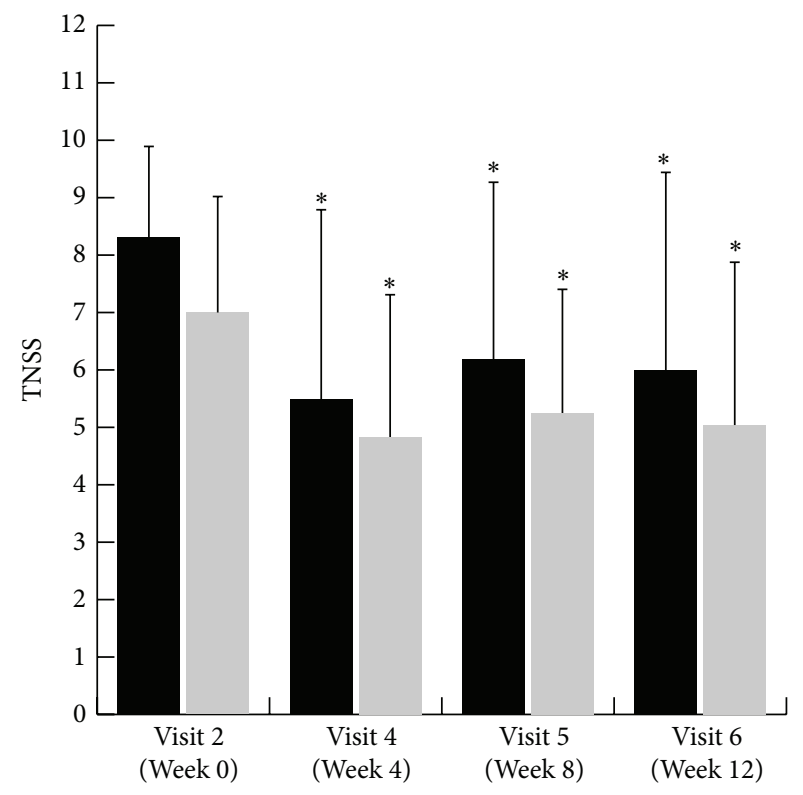

AR
NAR

(a)

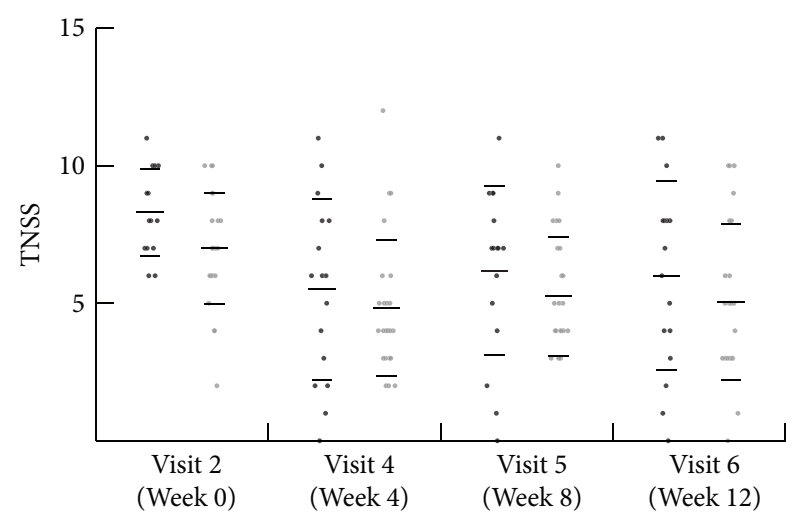

(c)

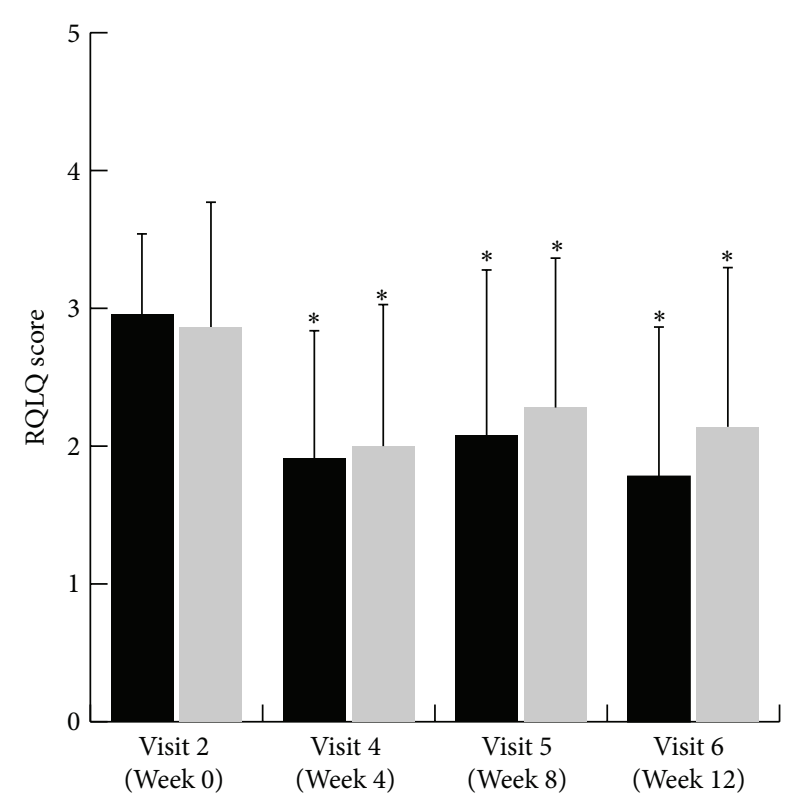

ar

NAR

(b)

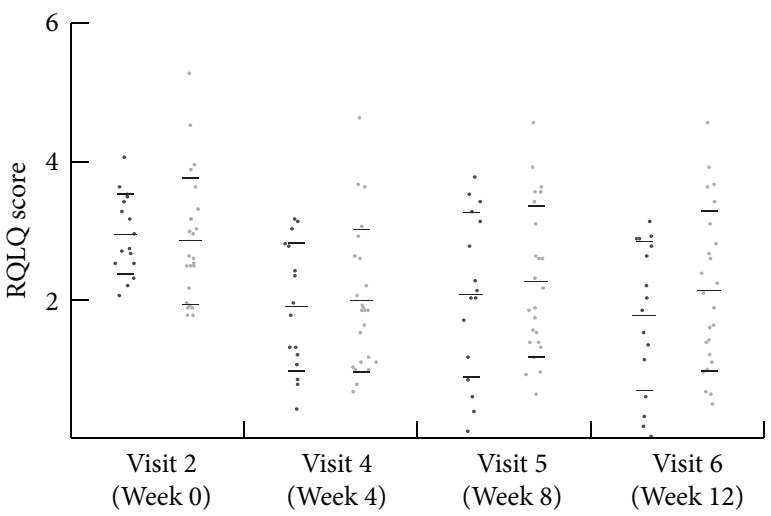

(d)

FIGURE 1: Effects of HYT on the AR group and the NAR group. (a and c) Mean TNSS at each visit. (b and d) Mean RQLQ score at each visit. * Significant difference $(p<0.05)$ compared with week 0 (baseline) in each group (repeated-measures ANOVA test and Bonferroni post hoc test). HYT, Hyeonggaeyeongyo-tang; AR, allergic rhinitis; NAR, nonallergic rhinitis; TNSS, total nasal symptom score; RQLQ, Rhinoconjunctivitis Quality of Life Questionnaire.

difference in any of the nasal symptoms between the AR group and the NAR group (Table 5).

3.3. RQLQ. The RQLQ showed a statistically significant decrease after medication and this improvement lasted for follow-up period in the whole subject group (CR), the AR group, and the NAR group. There was no significant betweengroup difference at any time point (Table 6 and Figures 1(b) and $1(\mathrm{~d}))$.

3.4. Serum Total IgE and Eosinophil Count. There was no significant change in total serum IgE or eosinophil count after treatment in the AR group (Table 7).
3.5. Safety. There were no serious adverse events observed or reported during the study. The only minor adverse events observed during treatment were dyspepsia $(n=3)$ and xerostomia $(n=2)$, but these events disappeared during the follow-up period. There were statistically significant differences in BUN, RBC, hemoglobin, and hematocrit after treatment. However, all these changes were in normal ranges and there was no subject who showed abnormal value after treatment assessment (Table 8).

\section{Discussion}

CR is chronic form of rhinitis and has been classified as AR and NAR. Approximately 50\% of CR patients are classified 
TABLE 5: Nasal symptom score from baseline to week 4 in AR and NAR group.

\begin{tabular}{lccc}
\hline & Week 0 & Week 4 & $p$ value \\
\hline Rhinorrhea & & & \\
$\quad$ AR & $2.06 \pm 0.93$ & $1.44 \pm 1.09$ & $0.036^{*}$ \\
$\quad$ NAR & $1.88 \pm 0.90$ & $1.29 \pm 0.95$ & $0.001^{*}$ \\
Nasal congestion & & & \\
$\quad$ AR & $1.69 \pm 0.95$ & $1.00 \pm 0.89$ & $0.029^{*}$ \\
NAR & $1.92 \pm 0.88$ & $1.42 \pm 0.72$ & $0.043^{*}$ \\
Nasal itching & & & \\
$\quad$ AR & $2.50 \pm 0.63$ & $1.56 \pm 1.03$ & $0.001^{*}$ \\
$\quad$ NAR & $1.54 \pm 0.98$ & $0.92 \pm 1.02$ & $0.002^{*}$ \\
Sneezing & & & \\
$\quad$ AR & $2.06 \pm 0.44$ & $1.50 \pm 0.97$ & $0.045^{*}$ \\
NAR & $1.67 \pm 0.96$ & $0.96 \pm 0.86$ & $0.001^{*}$ \\
\hline
\end{tabular}

Mean \pm standard deviation and paired $t$-test.

* Significant difference $(p<0.05)$ between week 0 (baseline) and week 4 in each group.

TNSS, total nasal symptom score; AR, allergic rhinitis; NAR, nonallergic rhinitis.

TABLE 6: Mean RQLQ scores from baseline to week 12.

\begin{tabular}{lcccc}
\hline & Week 0 & Week 4 & Week 8 & Week 12 \\
\hline All subjects & $2.90 \pm 0.79$ & $1.96 \pm 0.98^{*}$ & $2.20 \pm 1.12^{*}$ & $2.00 \pm 1.13^{*}$ \\
AR & $2.96 \pm 0.58$ & $1.91 \pm 0.93^{*}$ & $2.08 \pm 1.19^{*}$ & $1.78 \pm 1.08^{*}$ \\
NAR & $2.86 \pm 0.91$ & $2.00 \pm 1.03^{*}$ & $2.28 \pm 1.09^{*}$ & $2.14 \pm 1.16^{*}$ \\
\hline
\end{tabular}

Mean \pm standard deviation, repeated-measures ANOVA test, and Bonferroni post hoc test.

${ }^{*}$ Significant difference $(p<0.05)$ compared with week 0 (baseline) in each group (no significant difference between week 4 , week 8 , and week 12 in each group).

RQLQ, Rhinoconjunctivitis Quality of Life Questionnaire; AR, allergic rhinitis; NAR, nonallergic rhinitis.

TABLE 7: Total serum IgE and eosinophil count in the AR group.

\begin{tabular}{lccc}
\hline & Week 0 & Week 4 & $p$ value \\
\hline Total $\mathrm{IgE}(\mathrm{IU} / \mathrm{mL})$ & $287.93 \pm 321.26$ & $233.21 \pm 183.23$ & 0.486 \\
Eosinophil count $(/ \mu \mathrm{L})$ & $271.25 \pm 211.03$ & $281.25 \pm 244.29$ & 0.847 \\
\hline
\end{tabular}

Mean \pm standard deviation and paired $t$-test.

IgE, immunoglobulin E; AR; allergic rhinitis.

as having $\mathrm{AR}$, and the others are classified as having NAR [13]. AR is characterized by a specific IgE response against relevant aeroallergens. NAR encompasses all forms of rhinitis in which a specific IgE response against relevant aeroallergens is absent.

The main medications currently used for allergic and nonallergic rhinitis are antihistamines, nasal steroids, nasal decongestants, and leukotriene receptor antagonists $[14,15]$. In many cases of CR, symptoms are prolonged for years throughout all seasons; therefore, it is necessary to find the medicine which has no adverse effects for long-term medication and has long lasting effects. However, long-term use of many of the medications used for the treatment of CR can cause adverse effects. Antihistamines are the most widely used treatment for both AR and NAR. Antihistamines have limited efficacy in treating nasal congestion and commonly cause adverse effects such as sedation and weight gain [16, 17]. Nasal decongestants are useful for nasal obstruction, but using them for more than a week is not recommended because of adverse effects and drug tolerance [18]. For these reasons, traditional Chinese medicines which are made with natural herbs have recently gained much interest [19].

In this study, HYT improved nasal symptoms and quality of life in patients with AR and NAR after 4 weeks of medication, and these effects lasted 8 weeks after the end of medication. Each rhinitis patient has different symptoms based on his or her type of rhinitis and environmental factors. Some patients mainly have watery rhinorrhea and sneezing symptoms, while others mainly have nasal obstruction symptoms. In our study, both rhinorrhea and nasal obstruction were improved in patients with AR and NAR.

Other studies reported that HYT has anti-inflammation effects for AR by the suppression of NF- $\kappa \mathrm{B}$ activation and iNOS production in $\mathrm{BALB} / \mathrm{c}$ mice [9]. In several previous studies, main components of HYT, Schizonepeta tenuifolia, Forsythia fruit, Saposhnikovia divaricata, and Bupleurum falcatum, were observed to have antioxidant and antiinflammatory activities [20-23]. These antioxidant and antiinflammatory activities could be the reason of the effect of HYT in patients with AR and NAR.

In a previous animal study, HYT had antiallergic effects, inhibiting the increase of the levels of IL-4, IL-13, leukemia inhibitory factor (LIF), eosinophils, neutrophils, monocytes, basophils, lymphocytes, and WBC in ovalbumin-induced AR model [10]. Based on previous studies, we hypothesized that IgE and eosinophils will be suppressed in patients with AR; however, current study showed no decrease in $\operatorname{IgE}$ and eosinophils in AR patients. By referring to previous clinical study that reported changes of serum IgE, cytokines, IL-4 stimulated prostaglandin E2 (PGE2), and polymorphonuclear leukocyte (PMN) after 12-week administration of oriental herbal medicine, duration of medication needed to be longer in our study to observe the changes of serum $\operatorname{IgE}$ and eosinophils $[24,25]$.

There were statistically significant differences in BUN, RBC, hemoglobin, and hematocrit after treatment. However, all these changes were in normal ranges and there was no subject who showed abnormal value after treatment assessment. A clinical study with long-term medication for at least 8 weeks and the safety assessment would be necessary to confirm the long-term safety of HYT and to observe antiallergic effects in laboratory tests.

Our study has several limitations. First, this study is prepost study that was conducted with a nonrandomized and nocontrol-group design. Second, our sample size was relatively small. Third, the medication period was relatively short for safety assessment.

Despite these limitations, this study has a meaning as the first clinical study for HYT in CR. From this study, we suggest that HYT could be investigated as a medicine for patients with AR and NAR. A clinical study with a randomized, double-blind, placebo-controlled design, larger sample size, and long-term medication should be performed to obtain 
TABLE 8: The laboratory tests for safety assessment.

\begin{tabular}{|c|c|c|c|c|}
\hline & Normal ranges & Week 0 & Week 4 & $p$ value \\
\hline AST & $0 \sim 40(\mathrm{U} / \mathrm{L})$ & $21.50 \pm 6.84$ & $24.45 \pm 14.47$ & 0.148 \\
\hline ALT & $0 \sim 40(\mathrm{U} / \mathrm{L})$ & $18.84 \pm 11.39$ & $22.95 \pm 22.43$ & 0.167 \\
\hline BUN & $8 \sim 23(\mathrm{mg} / \mathrm{dL})$ & $13.24 \pm 3.94$ & $11.73 \pm 3.26$ & $0.005^{*}$ \\
\hline Creatinine & $0.6 \sim 1.2(\mathrm{mg} / \mathrm{dL})$ & $0.74 \pm 0.15$ & $0.73 \pm 0.16$ & 0.354 \\
\hline \multicolumn{5}{|l|}{$\mathrm{CBC}$} \\
\hline WBC & $4.0 \sim 10.0\left(\times 10^{3} / \mu \mathrm{L}\right)$ & $6.76 \pm 1.33$ & $6.45 \pm 1.38$ & 0.116 \\
\hline $\mathrm{RBC}$ & $4.2 \sim 6.3\left(\times 10^{6} / \mu \mathrm{L}\right)$ & $4.66 \pm 0.51$ & $4.57 \pm 0.48$ & $0.019^{*}$ \\
\hline Hemoglobin & $13 \sim 17(\mathrm{~g} / \mathrm{dL})$ & $13.93 \pm 1.60$ & $13.64 \pm 1.53$ & $0.002^{*}$ \\
\hline Hematocrit & $36 \sim 48(\%)$ & $41.64 \pm 4.24$ & $40.87 \pm 4.01$ & $0.011^{*}$ \\
\hline Platelet & $150 \sim 350\left(\times 10^{3} / \mu \mathrm{L}\right)$ & $261.95 \pm 46.21$ & $261.49 \pm 51.24$ & 0.890 \\
\hline ESR & $\sim 20(\mathrm{~mm} / \mathrm{h})$ & $12.62 \pm 8.97$ & $11.49 \pm 8.30$ & 0.290 \\
\hline
\end{tabular}

Mean \pm standard deviation and paired $t$-test.

${ }^{*}$ Significant difference $(p<0.05)$ between week 0 (baseline) and week 4 .

AST, aspartate transaminase; ALT, alanine transaminase; BUN, blood urea nitrogen; CBC, complete blood count; WBC, white blood cell; RBC, red blood cell; ESR, erythrocyte sedimentation rate.

more accurate knowledge about HYT treatment for rhinitis and to observe its mechanism.

\section{Competing Interests}

The authors declare that there are no competing interests regarding the publication of this paper.

\section{Authors' Contributions}

Min-Hee Kim and Jaewoong Son contributed equally to this work.

\section{Acknowledgments}

This study was funded by a grant from the Traditional Korean Medicine R\&D Project, Ministry of Health \& Welfare, Republic of Korea (HI12C1889 and HI13C0530).

\section{References}

[1] E. Middleton Jr., "Chronic rhinitis in adults," Journal of Allergy and Clinical Immunology, vol. 81, no. 5, pp. 971-975, 1988.

[2] M. F. Mullarkey, J. S. Hill, and D. R. Webb, "Allergic and nonallergic rhinitis: their characterization with attention to the meaning of nasal eosinophilia," The Journal of Allergy and Clinical Immunology, vol. 65, no. 2, pp. 122-126, 1980.

[3] R. A. Settipane and P. Lieberman, "Update on nonallergic rhinitis," Annals of Allergy, Asthma \& Immunology, vol. 86, no. 5, pp. 494-507, 2001.

[4] C. Bachert, P. van Cauwenberge, J. Olbrecht, and J. van Schoor, "Prevalence, classification and perception of allergic and nonallergic rhinitis in Belgium," Allergy, vol. 61, no. 6, pp. 693-698, 2006.

[5] B.-H. Jang, I.-H. Choi, K.-S. Kim et al., "Current status of allergic rhinitis patients in Korean Medicine hospitals-exploratory study based on electronic medical records of 3 hospitals," The Journal of Korean Medical Ophthalmology \& Otolaryngology \& Dermatology, vol. 27, no. 1, pp. 117-129, 2014.
[6] N.-K. Kim, D.-H. Lee, E.-S. Seo et al., “Treatment packages of persistent allergic rhinitis for developing PRCT protocols: an expert survey," The Journal of Oriental Medical Preventive, vol. 17, no. 3, pp. 143-153, 2013.

[7] T.-S. Yu, Y.-S. Jin, and G.-M. Jeong, "Study of the effects of Hyunggaeyeungyotang on the Anti-allergic effect in rats and mice," The Journal of Korean Oriental Pediatrics, vol. 4, no. 1, pp. 19-30, 1990.

[8] E.-J. Park and S.-Y. Shin, "Effects of Hyunggyeyungyotang and Kamihyunggyeyungyotang administration on the antiinflammation, analgesia and anti-allergic reaction in mice," The Journal of Korean Oriental Pediatrics, vol. 11, no. 1, pp. 249-273, 1997.

[9] J.-H. Park and S.-U. Hong, "The effects of hyunggaeyungyotang of suppression of iNOS production on mice with allergic rhinitis," The Journal of Korean Medicine Ophthalmology and Otolaryngology and Dermatology, vol. 25, no. 1, pp. 12-21, 2012.

[10] S. H. Hong, S. R. Kim, H.-S. Choi et al., "Effects of hyeonggaeyeongyo-tang in ovalbumin-induced allergic rhinitis model," Mediators of Inflammation, vol. 2014, Article ID 418705, 9 pages, 2014.

[11] K. S. Kim and Y.-B. Kim, "Anti-inflammatory effect of Keigairengyo-to extract and acupuncture in male patients with acne vulgaris: a randomized controlled pilot trial," The Journal of Alternative and Complementary Medicine, vol. 18, no. 5, pp. 501508, 2012.

[12] J. Bousquet, N. Khaltaev, A. A. Cruz et al., "Allergic rhinitis and its impact on asthma (ARIA) 2008," Allergy, vol. 63, supplement 86, pp. 8-160, 2008.

[13] T. L. Smith, "Vasomotor rhinitis is not a wastebasket diagnosis," Archives of Otolaryngology-Head \& Neck Surgery, vol. 129, no. 5, pp. 584-587, 2003.

[14] E. O. Meltzer, M. S. Blaiss, M. J. Derebery et al., "Burden of allergic rhinitis: results from the Pediatric Allergies in America survey," Journal of Allergy and Clinical Immunology, vol. 124, no. 3, pp. S43-S70, 2009.

[15] G. Ciprandi, "Treatment of nonallergic perennial rhinitis," Allergy, vol. 59, no. 76, pp. 16-23, 2004.

[16] M. K. Church, M. Maurer, C. Bindslev-Jensen et al., "Risk of first-generation $\mathrm{H}_{1}$-antihistamines: a GA ${ }^{2} \mathrm{LEN}$ position paper," Allergy, vol. 65, no. 4, pp. 459-466, 2010. 
[17] F. E. R. Simons, "H1-Antihistamines: more relevant than ever in the treatment of allergic disorders," Journal of Allergy and Clinical Immunology, vol. 112, no. 4, pp. S42-S52, 2003.

[18] P. Graf, "Adverse effects of benzalkonium chloride on the nasal mucosa: allergic rhinitis and rhinitis medicamentosa," Clinical Therapeutics, vol. 21, no. 10, pp. 1749-1755, 1999.

[19] R. Y. P. Chan and W. T. Chien, "The effects of two Chinese herbal medicinal formulae vs. placebo controls for treatment of allergic rhinitis: a randomised controlled trial," Trials, vol. 15, article 261, 2014.

[20] B.-S. Wang, G.-J. Huang, H.-M. Tai, and M.-H. Huang, "Antioxidant and anti-inflammatory activities of aqueous extracts of Schizonepeta tenuifolia Briq," Food and Chemical Toxicology, vol. 50, no. 3-4, pp. 526-531, 2012.

[21] H. S. Kang, J. Y. Lee, and C. J. Kim, "Anti-inflammatory activity of arctigenin from Forsythiae Fructus," Journal of Ethnopharmacology, vol. 116, no. 2, pp. 305-312, 2008.

[22] J. M. Chun, H. S. Kim, A. Y. Lee, S. Kim, and H. K. Kim, "Anti-inflammatory and antiosteoarthritis effects of saposhnikovia divaricata ethanol extract: in vitro and in vivo studies," Evidence-Based Complementary and Alternative Medicine, vol. 2016, Article ID 1984238, 8 pages, 2016.

[23] W. H. Park, S. Kang, Y. Piao et al., "Ethanol extract of Bupleurum falcatum and saikosaponins inhibit neuroinflammation via inhibition of NF- $\kappa$ B,' Journal of Ethnopharmacology, vol. 174, pp. 37-44, 2015.

[24] S.-H. Yang, C.-L. Yu, Y.-L. Chen, S.-L. Chiao, and M.-L. Chen, "Traditional Chinese medicine, Xin-yi-san, reduces nasal symptoms of patients with perennial allergic rhinitis by its diverse immunomodulatory effects," International Immunopharmacology, vol. 10, no. 8, pp. 951-958, 2010.

[25] S.-H. Yang and C.-L. Yu, "Antiinflammatory effects of Buzhong-yi-qi-tang in patients with perennial allergic rhinitis," Journal of Ethnopharmacology, vol. 115, no. 1, pp. 104-109, 2008. 


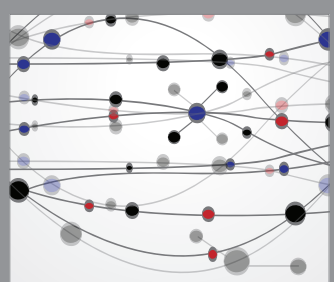

The Scientific World Journal
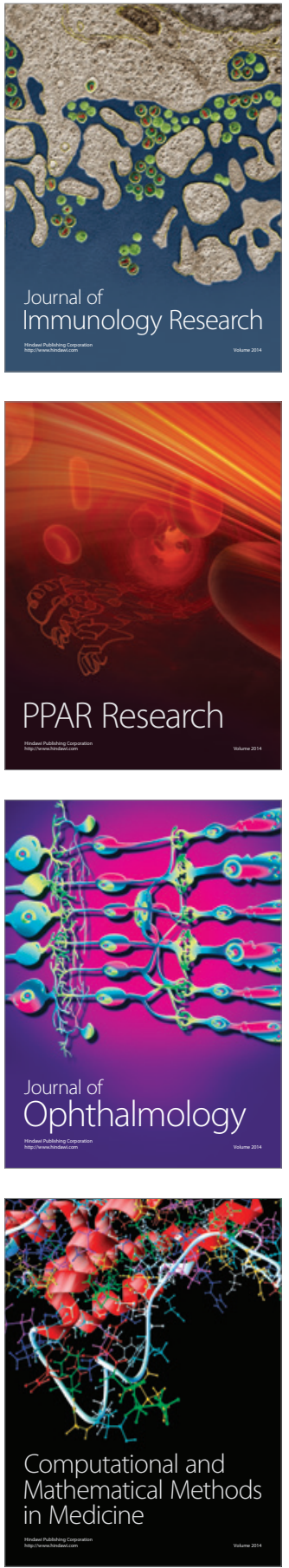

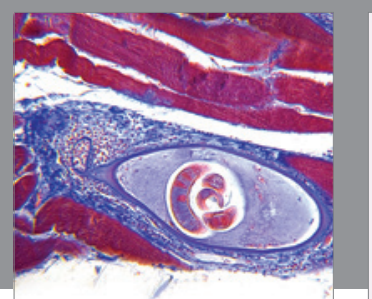

Gastroenterology Research and Practice

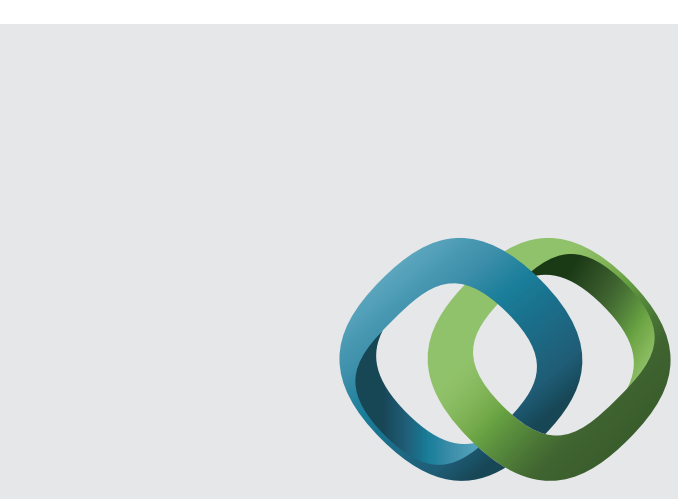

\section{Hindawi}

Submit your manuscripts at

http://www.hindawi.com
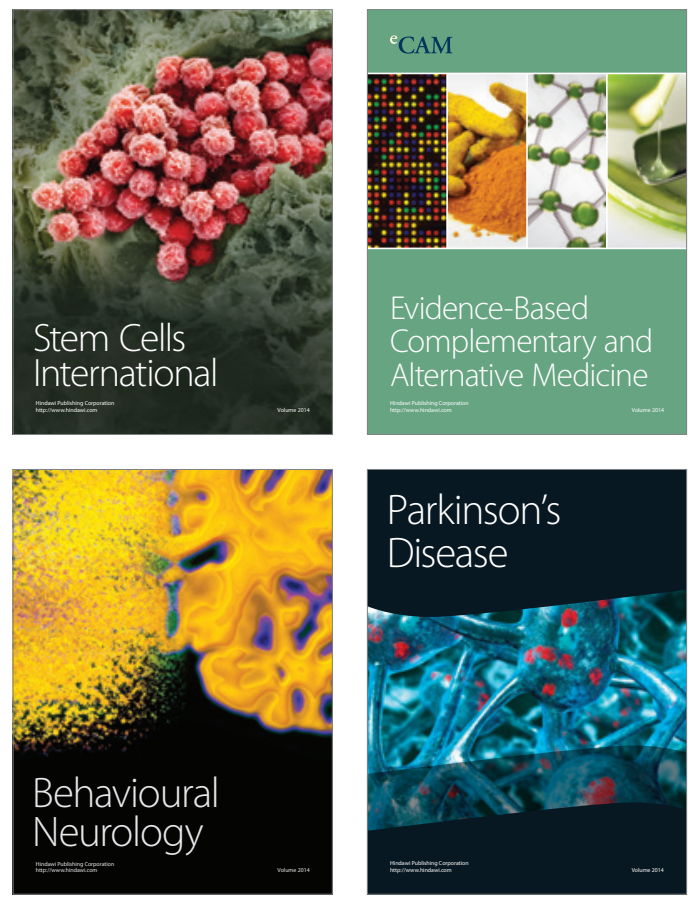
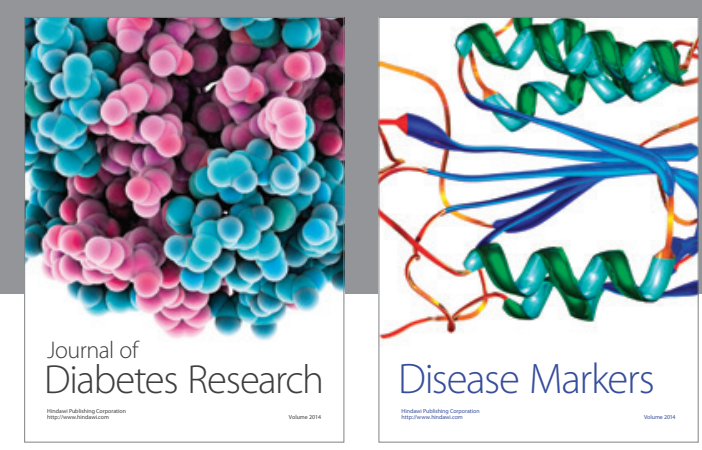

Disease Markers
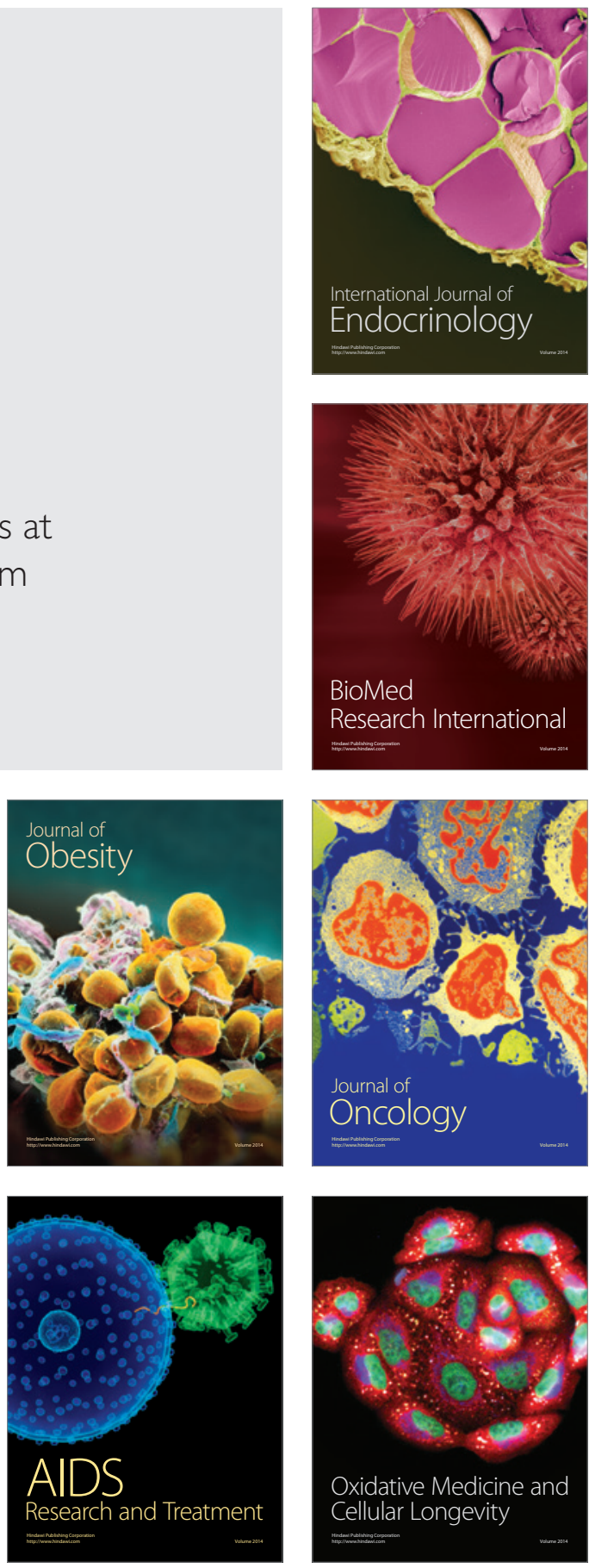